\title{
A Role for buttonhead in the Early Head and Trunk Development in the Beetle Tribolium castaneum
}

\author{
Haewon Jeon ${ }^{1}$, Jiyun $\mathrm{O}^{1}$, Sil Jin' ${ }^{1}$ Jinsung Lim²$^{2}$, and ${ }^{\dagger}$ Chong Pyo Choe ${ }^{1,2}$

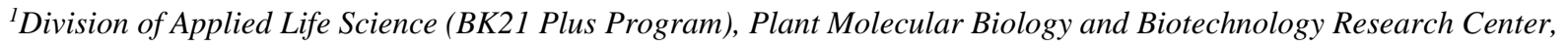 \\ Gyeongsang National University, Jinju 52828, Korea \\ ${ }^{2}$ Division of Life Science, Gyeongsang National University, Jinju 52828, Korea
}

\begin{abstract}
The head gap gene buttonhead $(b t d)$ is required for the patterning of head segments in the early Drosophila embryo. Mutant phenotypes of btd display a gap-like phenotype in which antennal, intercalary, mandibular and the anterior portion of the maxillary segments are eliminated. In agreement with the phenotypes, btd is expressed in a stripe covering the head segments at the blastoderm stage. During the early phase of the germband extension, btd is expressed in stripes with single segmental periodicity, which is required for the formation of the peripheral nervous system. In contrast to the key role of btd in Drosophila embryonic development, it has been suggested that Tribolium ortholog of btd (Tc-btd) is dispensable for embryonic head development. In order for better understanding of the requirement of Tc-btd in the early Tribolium embryo, we re-analyzed the expression patterns and functions of $T c$-btd during embryonic segmentation. Tc-btd is expressed in segmental stripes at the stages of blastoderm and germband elongation. Up to $28.3 \%$ of embryos in which $T c$-btd is knocked down displays the loss of antennal, mandibular and the pregnathal regions in the head, with abdominal segments being disrupted in the trunk. Our findings suggest that $T c$-btd is required for the head and trunk development in the early Tribolium embryo.
\end{abstract}

Key words : buttonhead, Head gap gene, Segmentation, Tribolium

\section{INTRODUCTION}

During embryonic head development, all insects build segments that fuse to each other to form the head capsule bearing appendages with different roles in feeding and feeling (Snodgrass, 1935). The insect head consists of the anterior pregnathal and the posterior gnathal regions. While the anterior pregnathal region comprises the intercalary (IC), antennal (AN), ocular segments (OC), and other preantennal tissues, the posterior gnathal region is composed of the mandibular (MD), maxillary (MX), and labial (LB) segments that constitute the mouthparts (Snodgrass, 1935; Rogers and Kaufman, 1997). The genetic mechanisms of head segmentation have been studied in most detail in Drosophila melanogaster. In Drosophila, the typical hierarchy of maternal, gap, pair-rule, and segment polarity gene interactions form the maxillary and labial gnathal segments (St Johnston \& Nusslein-Volhard, 1992). For patterning the pregnathal and the mandibular segments, however, Drosophila utilizes the head gap genes, orthodenticle (otd), empty spiracles (ems) and buttonhead $(b t d)$, which are expressed in broad and overlapping do-

\footnotetext{
Manuscript received January 9, 2019, Received in revised form January 30, 2019, Accepted February 22, 2019

${ }^{\dagger}$ Corresponding Author : Chong Pyo Choe, Division of Life Science, Gyeongsang National University, Jinju 52828, Korea. Tel: +82-55-772-1367, E-mail: cpchoe@gnu.ac.kr

${ }^{*}$ The authors declare that they have no conflict of interest.

This is an Open Access article distributed under the terms of the Creative Commons Attribution Non-Commercial License (http:// creative-commons.org/licenses/by-nc/3.0) which permits unrestricted non-commercial use, distribution, and reproduction in any medium, provided the original work is properly cited.
} 
mains of the embryonic head region (Dalton et al., 1989; Cohen \& Jurgens, 1990; Finkelstein \& Perrimon, 1990; Walldorf \& Gehring, 1992; Wimmer et al., 1993). Consistently, mutations in any head gap gene display phenotypes missing the pregnathal and the mandibular segments that correspond to the expression domains of each head gap gene. For example, otd mutants do not bear the antennal and ocular segments, and ems mutants do not have the antennal, intercalary, and the anterior portion of the mandibular segments (Dalton et al., 1989; Cohen \& Jurgens, 1990; Walldorf \& Gehring, 1992). In addition, loss of btd leads to the lack of antennal, intercalary, mandibular and the anterior portion of the maxillary segments (Wimmer et al., 1996).

It has been suggested that the genetic mechanisms of head segmentation in Drosophila might not be always applicable to other insect head segmentation. In Drosophila, activation of the head gap genes depends on Bicoid, which is the key maternal factor for patterning anterior regions along the anterior-to-posterior axis of Drosophila embryo (Finkelstein \& Perrimon, 1990; Wimmer et al., 1995; Gao \& Finkelstein, 1998). Interestingly, however, it has become clear that bicoid is absent from most other insects but probably arose recently in the lineage of dipterans during insect evolution (Stauber et al., 1999; Schroder, 2003; Chouard, 2008). Furthermore, Drosophila larva has the derived head morphology including the maggot's head being hidden within the thorax and the head structures being highly derived (Rogers \& Kaufman, 1996). To understand the evolution of genetic mechanisms controlling head development, orthologs of Drosophila head gap genes have been analyzed in insects (Schinko et al., 2008). In the red flour beetle Tribolium castaneum, functional analyses of orthologs of Drosophila head gap genes revealed that the head segmentation mechanisms in Tribolium are distinct from those of Drosophila. Similar to Drosophila, Tcotdl plays as a head gap gene for the early regionalization of all head segments formed during the blastoderm stage and then for the later head patterning (Schinko et al., 2008). However, it has been suggested that Tc-ems and Tcbtd are not head gap genes; Tc-ems has a minor role for patterning the antennal segment and Tc-btd is not required for head development at all (Schinko et al., 2008). The dispensable role for Tc-btd in Tribolium head development is unexpected and remains arguable because $T c$-btd is expressed in the future mandibular segment at the blastoderm stage, with its later expression in the antennal and intercalary segments (Schinko et al., 2008). In the milkweed bug Oncopeltus fasciatus, knock-down of Of-otd leads to the lack or reduction of the anterior region to the mandibular segment, with the mandibular and maxillary segments being malformed (Birkan et al., 2011). However, roles for Of-ems could not be analyzed because of the very low penetrance of RNAi effects (Birkan et al., 2011).

Here we reexamined the expression patterns and functions of Tc-btd during Tribolium segmentation for better understanding the role of Tc-btd in Tribolium development. Tc-btd is expressed in stripes with single segmental periodicity at the segmentation stages, with its later expression in the head segments and head lobes. Larval cuticles of $T c$ - $b t d$ RNAi (Tc-btd ${ }^{\mathrm{RNAi}}$ ) embryos display the loss of pregnathal regions with the gnathal maxillary and labial segments remaining in the head and the defects in abdominal segmentation in the trunk. The defects are observed in the head and trunk regions of the growing germbands undergoing embryonic segmentation. Our observations suggest that $T c$-btd is required for the head and trunk development in Tribolium.

\section{MATERIALS AND METHODS}

\section{Isolation of Tribolium btd cDNA}

Tc-btd sequence was identified in the Tribolium genome (GenBank accession number: NM_001114320; Tc number: TC011696). A cDNA fragment of Tc-btd spanning the Btd box and zinc finger domains was amplified by using 
PCR (Tc-btd_F: 5'-GTGACTACTATGATGGCT-3' and Tc-btd_R: 5'-TTGCTCCCCCAAAGTAAT-3') then cloned into the pGEM ${ }^{\circledR}$-T Easy Vector (Promega). The cDNA sequence is available in Genbank under the accession number of MK546416.

\section{Sequence alignment and phylogenetic analysis}

Phylogenetic analysis was carried out using the amino acid sequences of insect orthologs of Btd obtained from Genbank: (Drosophila-Btd, Drosophila melanogaster NP_ 511100; Anopheles-Btd, Anopheles gambiae XP_311200; Drosophila-Sp1, Drosophila melanogaster NP_727360; Anopheles- Sp1, Anopheles gambiae XP_311199; Tribolium-Sp8, Tribolium castaneum NP_001034509; HumanSP1, Homo sapiens NM_001251825). CLUSTAL Omega with default parameters was used to align the CDS of TcBtd with other Btd/Sp orthologs (Sievers et al., 2011). A maximum likelihood tree was constructed using the PhyML method with default parameters (Lefort et al., 2017).

\section{Whole mount in situ hybridization and immuno-} chemistry

Whole-mount in situ hybridization was performed as described with some modifications (Kim et al., 2013). The digoxigenin-labeled $T c$-btd riboprobe was transcribed with $1 \mu \mathrm{g}$ of the $\mathrm{pGEM}^{\circledR}-\mathrm{T}$ Easy Vector containing the $T c-b t d$ cDNA fragment using T7 RNA polymerase (Ambion). Wild-type (GA2) embryos were collected in 24 hours-postcrossing, which were temporally heterogeneous. The embryos were dechorionated in $50 \%$ liquid chlorine bleach and then fixed for $15 \mathrm{~min}$ in $4 \%$ formaldehyde in Phosphate-Buffered Saline (PBS). The fixed embryos were dehydrated in $\mathrm{MeOH}$, rehydrated in PBT (PBS containing $0.1 \%$ Tween 20), then treated with xylene to increase permeability for the riboprobes. Embryos were hybridized with Tc-btd riboprobes in hybridization buffer (50\% formamide / 5X Saline Sodium Citrate Buffer (SSC) / yeast RNA at $200 \mu \mathrm{g} / \mathrm{mL} /$ heterologous DNA at $100 \mu \mathrm{g} / \mathrm{mL} /$
$0.1 \%$ Tween 20). The hybridized riboprobes were detected by Anti-Digoxigenin-AP (Roche) in conjunction with BM purple (Roche). Immunochemistry was performed as described previously with a 1:5 dilution of mAb anti-En (4D9) from the Developmental Studies Hybridoma Bank (DSHB) at the University of Iowa (Patel et al., 1994). Embryos that were stained with $T c-b t d$ riboprobe or anti-En (4D9) antibody were photographed on a BX50 compound microscope (Olympus, Melville, NY).

\section{RNAi analysis}

Parental RNAi for knocking down Tc-btd was performed as described (Bucher et al., 2002). A PCR fragment of Tc-btd that contains a $\mathrm{T} 7$ promoter on each end was generated with a T7-linked primer set (Tc-btd_T7F: 5'-TAATACGACTCACTATAGGGACCACCTGCACCA GGATGTAC-3' and $T c$ - $b t d$ T7R: 5'- TAATACGACTCA CTATAGGGTTCACCCCGTCACTGCTTTT-3'). dsRNA for $T c$-btd was synthesized with $1 \mu \mathrm{g}$ of PCR DNA template using T7 RNA polymerase (Ambion). $500 \mathrm{ng} / \mu \mathrm{L}$ or $750 \mathrm{ng} / \mu \mathrm{L}$ of $T c-b t d$ dsRNA were injected into wild-type (GA2) pupae. Injection buffer (0.1 mM Sodium Phosphate Buffer $\mathrm{pH} 6.8,5 \mathrm{mM} \mathrm{KCl}$ ) or $1 \mu \mathrm{g} / \mu \mathrm{L}$ of $T c$-fushi tarazu (FTZ) dsRNA was injected as a negative control and produced no defects in the head and trunk, as reported previously (Choe et al., 2006). Embryos were collected every 48 hours for 2 weeks and then incubated at $30^{\circ} \mathrm{C}$ for 24 hours or for 4 days to analyze the RNAi phenotypes.

\section{RESULTS}

\section{The Tribolium btd ortholog}

The Tribolium ortholog of btd was identified in the Tribolium genome. This predicted 846 bp of $T c-b t d$ that coded for a deduced protein of 282 amino acids. The deduced protein contained the C-X-C-P-X-C Btd box and $\mathrm{C} 2 \mathrm{H} 2$ zinc finger domains (Wimmer et al., 1993). A fragment of $T c$-btd containing the Btd box and zinc finger domains was 
amplified from wild-type cDNA (Fig. 1A). Alignment of the deduced 104 amino acid sequences with orthologs of $\mathrm{Btd} / \mathrm{Sp}$-family members from other insects displayed the well-conserved Btd box and zinc finger domains, which were highlighted in yellow and green, respectively in Fig. 1A. Zinc finger domains of Tribolium Btd showed 83.95\% identity with those of Anopheles and $69.62 \%$ identity with those of Drosophila. In addition, zinc finger domains of Tribolium Btd showed $91.36 \%$ identity with those of Tribolium Sp8, 92.59\% identity with those of Anopheles Sp1, and $92.59 \%$ identity with those of Drosophila Sp1. Phylogenetic tree constructed using maximum likelihood analysis from the complete deduced amino acid sequences of insect Btd/Sp-family members confirmed that the fragment of Btd box and zinc finger domains we isolated from Tribolium cDNA belonged to the insect btd gene (Fig. 1B).

\section{Expression patterns of Tc-btd in Tribolium seg-} mentation

Although the expression patterns of $T c$-btd during seg-

\section{A \\ Tribolium_Btd Drosophila_Btd Anopheles_Btd Tribolium_Sp8 Drosophi la_Sp1 Anopheles_Sp1 Human_SP1}

B

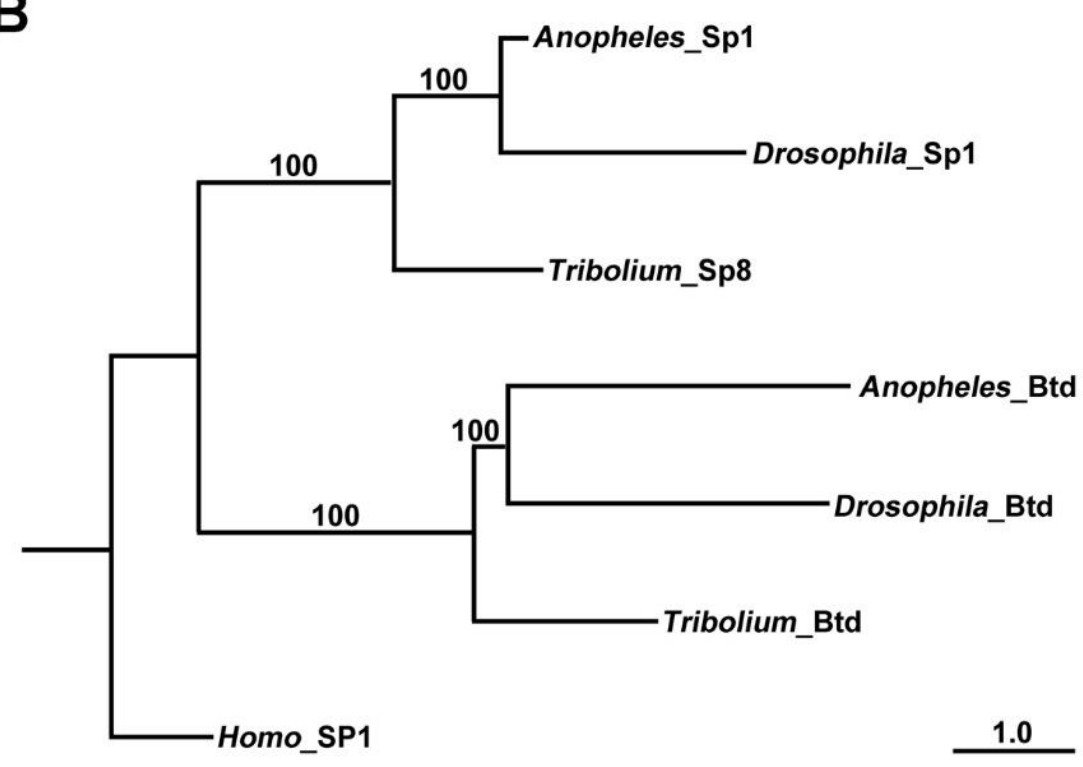

Fig. 1. The Tribolium ortholog of btd. (A) Alignment of the Btd box (highlighted in yellow) and zinc finger domains (highlighted in green) of Tc-Btd with those of insect Btd/Sp family members. They are highly conserved within insect orthologs of Btd/Sp family members. (B) Phylogenetic analysis of the complete deduced amino acid sequences of the Tribolium ortholog of btd. This rooted phylogenetic tree was constructed using maximum likelihood (ML) based on multiple sequence alignments of the complete deduced amino acid sequences of the insect orthologs of btd/sp using Homo sapiens SP1 as an outgroup. Bootstrap values are shown at branches. In this ML tree, Tribolium Btd is grouped with the group of Anopheles Btd and Drosophila Btd with a $100 \%$ of bootstrap value, whereas Tribolium Sp8 is with the group of Anopheles Sp1 and Drosophila Sp1 with a 100\% of bootstrap value. Tribolium, Tribolium castaneum; Drosophila, Drosophila melanogaster; Anopheles, Anopheles gambiae. 
mentation were reported previously in Tribolium (Schinko et al., 2008), we re-analyzed its expression patterns with our own riboprobe that hybridized with $629 \mathrm{bp}$ of Tc-btd mRNA. The first transcripts of $T c$-btd were detected as a stripe at approximately 50\% egg length from the posterior pole during the blastoderm stage (Fig. 2A). When the germ rudiment formed, the first stripe was located in the mandibular (MD) segment (Fig. 2B). As the germband grew, a series of $T c$-btd stripes appeared sequentially in the maxillary (MX), labial (LB), thoracic (T), and abdominal (A) segments, with their expression being maintained throughout the germband extension stages (Fig. 2C-H). When the Tc-btd stripe arose in the third thoracic (T3) segment, it appeared in the antennal (AN) segment (arrows in Fig. $2 \mathrm{E})$. As the germband grew, the antennal expression became stronger than its initial expression (arrows in Fig. $2 F)$. In the fully extended germband, new expression domains of $T c-b t d$ were observed in the pregnathal region (arrowheads in Fig. 2H). Compared to the early expression of btd in the future head regions of Drosophila embryos (Wimmer et al., 1993), Tc-btd expression in the head appeared in later stages of head development, as reported previously (Schinko et al., 2008). The late expression of $T c-b t d$ in the pregnathal region might imply a role for $T c$ $b t d$ in maintaining a segmental identity or promoting further differentiation of the head primordium, rather than a role in patterning the head segments per se. In addition, the segmental Tc-btd stripes in the thorax and abdomen of the growing germband suggest a role for $T c-b t d$ in the trunk segmentation.

\section{Defects in the head and abdomen of $T c-b t d^{\text {RNAi }}$ larva}

We investigated the role of $T c-b t d$ in the head and trunk segmentation using RNAi. First, we injected $500 \mathrm{ng} / \mu \mathrm{L}$ of Tc-btd dsRNA into wild-type (GA2) pupae. Among 127 cuticles of $T c-b t d^{\mathrm{RNAi}}$ larvae, $10.2 \%$ (13/127) showed defects in the head and trunk while the remaining $89.8 \%$ $(114 / 127)$ were normal. To increase RNAi efficiency, we

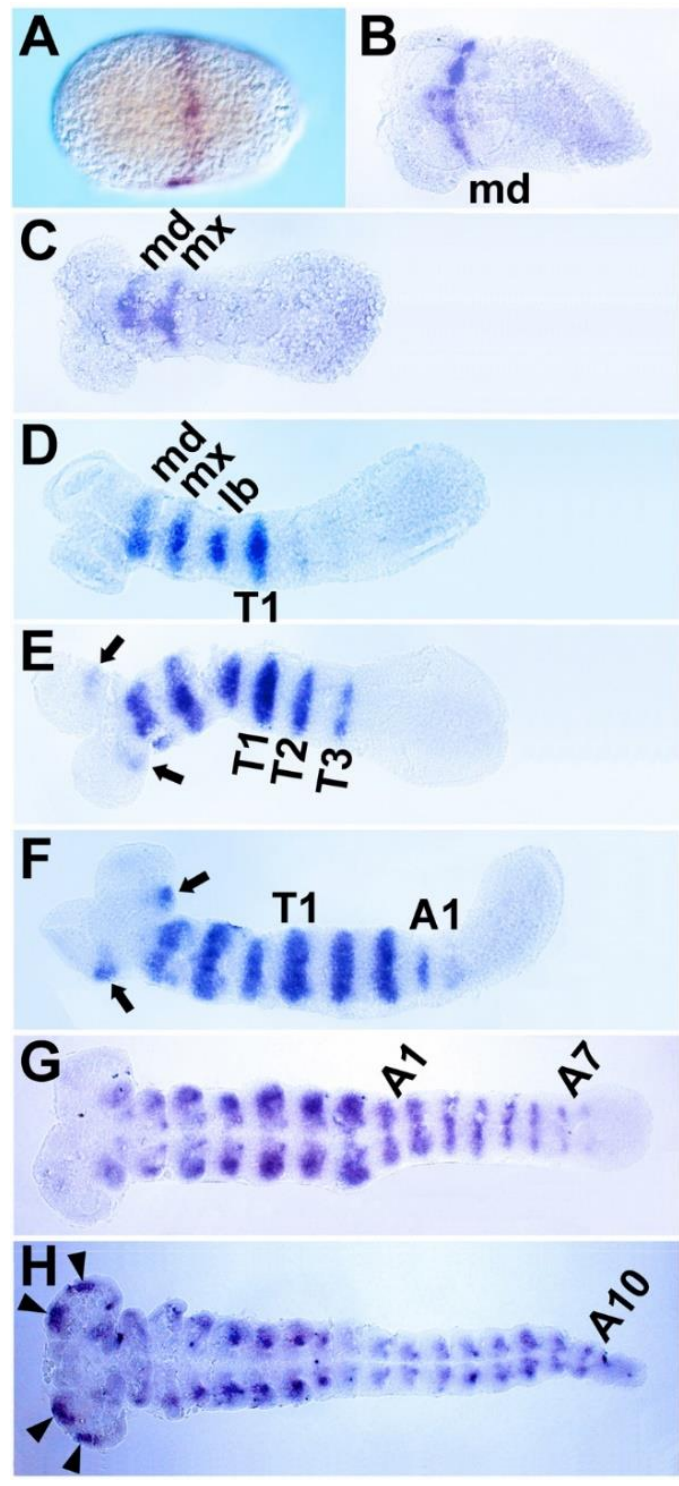

Fig. 2. In situ hybridization of Tc-btd in Tribolium embryonic segmentation. (A) A late stage of blastoderm embryo expressing $T c$-btd at approximately $50 \%$ egg length from the posterior pole. (B) An early germ rudiment that expresses $T c-b t d$ in the mandibular segment. (C-G) Elongating germbands that express stripes of $T c-b t d$ sequentially in the pregnathal, thoracic, and abdominal segments. While $T c$-btd is expressed in the trunk regions, it is not expressed in the posterior growth zone of each germband. Tc-btd expressions are seen in the antennal segments (arrows) in E and F. (H) A fully extended germband that completed segmentation. $T c$ $b t d$ expression in the head lobe is marked with arrowheads. Anterior is to the left. 
then injected $750 \mathrm{ng} / \mu \mathrm{L}$ of $T c-b t d$ dsRNA into wild-type (GA2) pupae. In this case, 28.3\% (49/173) showed similar defects in the head and trunk to those injected with 500 $\mathrm{ng} / \mu \mathrm{L}$ of $T c$-btd dsRNA. The remaining 71.7\% (124/173) showed normal head and trunk segmentation. Although the penetrance of $T c-b t d^{\mathrm{RNAi}}$ was quite low $(10.2 \%$ to $28.3 \%)$, $T c-b t d^{\mathrm{RNAi}}$ led to the reduced size of the head and the lack of several abdominal segments compared with wild types
(Fig. 3A, C). In most cases, the pregnathal region including the antenna (AN), and the mandible (MD) were lost and the maxillary (MX) was abnormally shaped in $T c$ $b t d^{\mathrm{RNAi}}$ larvae (Fig. 3B, D). However, the labium (LB) of $T c-b t d^{\mathrm{RNAi}}$ larvae appeared to be normal (Fig. 3B, D). Compared with wild types, in some cases, an uncharacterized head lobe formed on the dorsal of the thorax in addition to the reduced size of the head (Fig. 3E, F). As a nega-

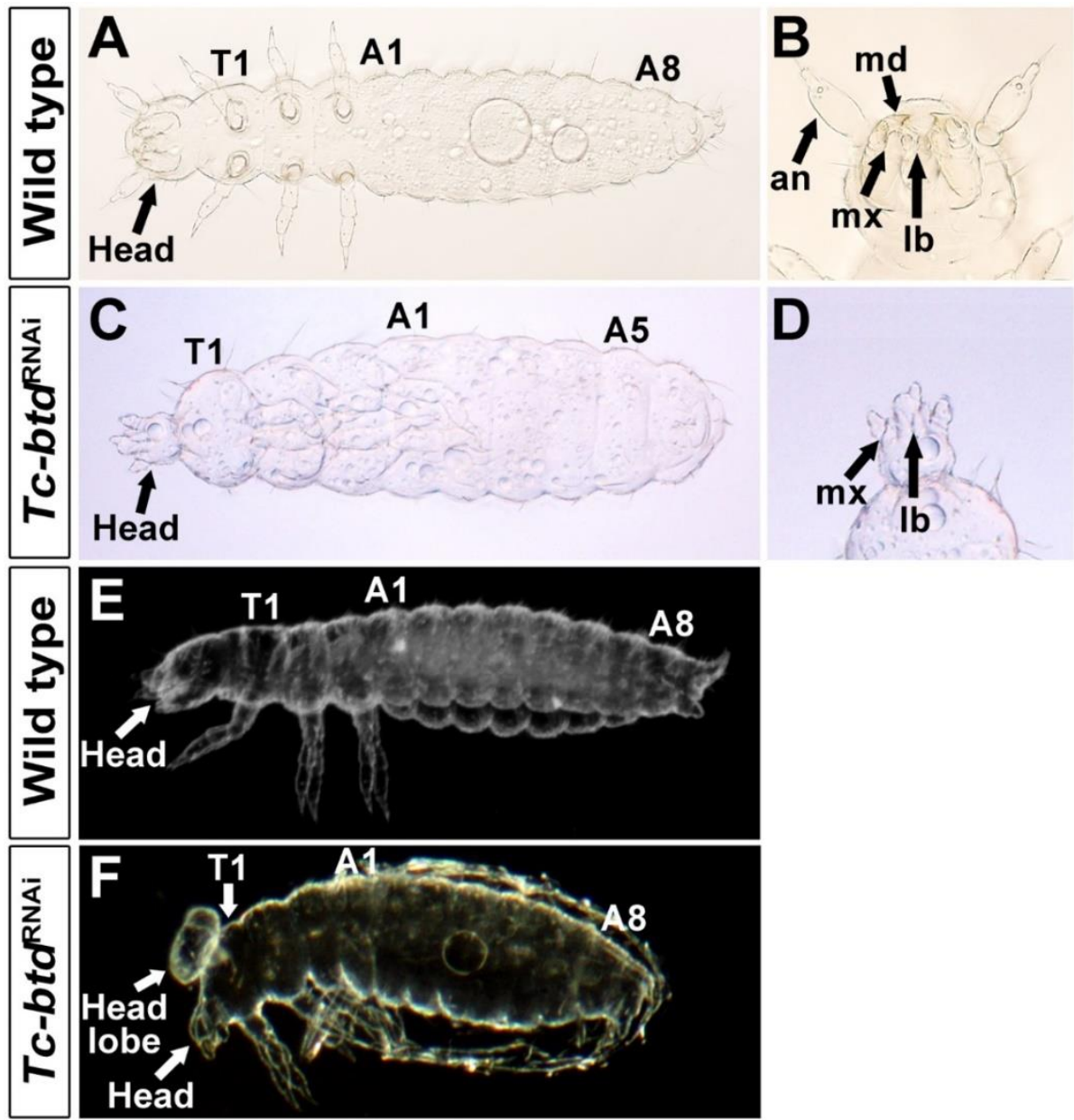

Fig. 3. Cuticle preparations in $T c$-btd ${ }^{\text {RNAi }}$ embryos. (A) Ventral view of wild-type larval cuticle with head, three thoracic segments bearing a pair of legs in each segment, and eight abdominal segments. (B) A magnified image of the wild-type head carrying the head appendages. (C) Ventral view of $T c$ - $b t d{ }^{\text {RNAi }}$ larva. This animal contains a small head, three thoracic, and five abdominal segments. (D) A zoom-in image of the small $T c-b t d{ }^{\text {RNAi }}$ larval head. The maxilla and labium are present, but the pregnathal regions are missing. (E) Lateral view of wild-type larval cuticle with head, three thoracic segments, and eight abdominal segments. (F) Lateral view of Tc-btd ${ }^{\text {RNAi }}$ larva. This animal has an uncharacterized head lobe on the dorsal of the thorax in addition to the small head. However, this animal develops normally three thoracic and eight abdominal segments. Note that the egg-shell is incompletely dissected from this late-stage embryo. (A, C, E, F) Anterior is to the left. (B, D) Anterior is to the top. 
tive control, $1 \times$ injection buffer or $1 \mu \mathrm{g} / \mu \mathrm{L}$ of $T c-f t z$ dsRNA, which was previously reported to have no effects on the segmentation of larval cuticles, was injected into wild-type (GA2) pupae (Choe et al., 2006). All cuticles of $1 \times$ injection buffer injected $(n=109)$ or Tc-ftz dsRNA injected $(n=146)$ larvae displayed the normal head and trunk. Although it was reported previously that $T c-b t d$ was dispensable for the development of head in Tribolium (Schinko et al., 2008), our results indicated the role of $T c$ $b t d$ in the trunk segmentation as well as head development.

\section{Germband defects in Tc-btd ${ }^{\text {RNAi }}$ embryos}

In order to verify the defects observed in the $T c-b t d^{\mathrm{RNAi}}$ larval cuticles, we examined embryonic segmentation process at the stage of germband extension. To do so, we analyzed expressions of Engrailed (Tc-En) in $T c-b t d^{\mathrm{RNAi}} \mathrm{em}$ bryos. In wild-type growing germbands, a series of Tc-En stripes arises sequentially in the gnathal, thoracic, and abdominal segments as well as in the antennal segment (Fig. 4A). In the growing germbands of $T c-b t d^{\mathrm{RNAi}}$ embryos, we observed irregular Tc-En stripes in the gnathal region, which was bifurcated (arrow in Fig. 4C). Interestingly, the fully extended germbands of $T c-b t d^{\mathrm{RNAi}}$ embryos that the segmentation was completed, were short and wide in addition to the irregular Tc-En stripes, compared with wild types (Fig. 4B, D). In particular, the head lobe including the pregnathal segments seemed to form abnormally or be absent in $T c$-btd ${ }^{\mathrm{RNAi}}$ germbands (arrows in Fig. 4D). In the same $T c-b t d^{\mathrm{RNAi}}$ embryo, we observed a trace of tissues (arrowhead in Fig. 4D'), which was anterior to the defective head region (arrow in Fig. 4D') before dissecting the germband from the yolk. Such a trace of tissues was never observed in wild-type embryos. The defects in the head and trunk of $T c-b t d^{\mathrm{RNAi}}$ germbands suggest that the abnormal head and abdomen of $T c-b t d^{\mathrm{RNAi}}$ larval cuticles might be caused by some defects occurred in the early segmenting germbands.

\section{DISCUSSION}

We have re-analyzed expression patterns and functions of Tc-btd in Tribolium segmentation. We confirm the segmental expression of $T c$-btd with single segmental periodicity during embryonic segmentation, as reported previ-

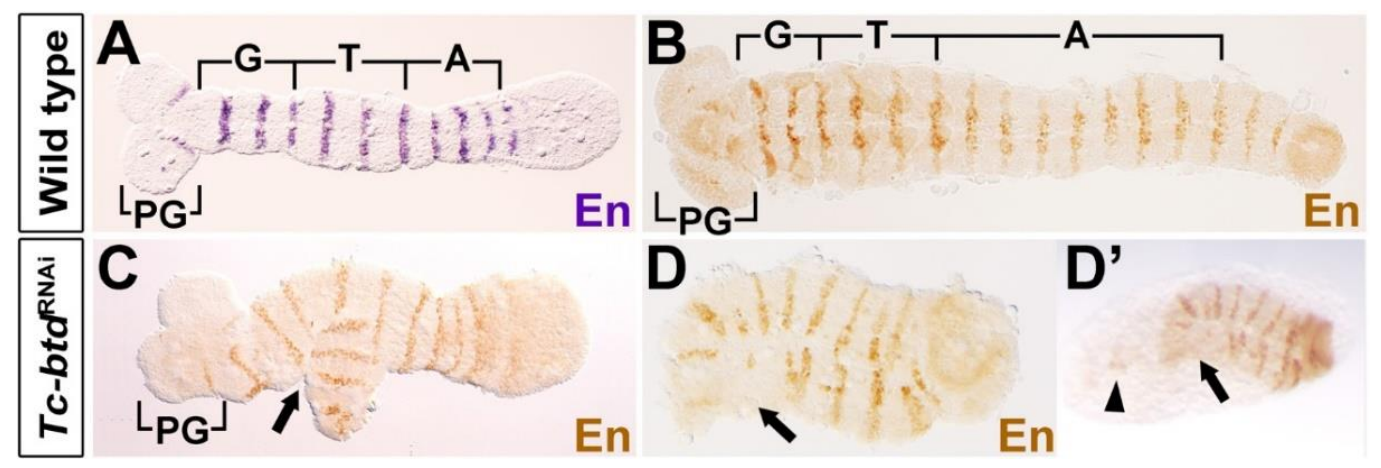

Fig. 4. Germbands of $\boldsymbol{T} \boldsymbol{c}$-btd ${ }^{\text {RNAi }}$ embryos. (A-D) Tribolium germbands stained with Anti-En antibody. (A) In the growing wild-type germband, Tc-En is expressed as stripes in the gnathal $(\mathrm{G})$, thoracic $(\mathrm{T})$ and abdominal $(\mathrm{A})$ regions. Tc-En is also seen in the antennal segment of the pregnathal (PG) region. (B) Tc-En is still expressed as stripes in the trunk of the fully grown wild-type germband. (C) This elongating $T c$-btd ${ }^{\text {RNAi }}$ germband has bifurcated gnathal regions (arrow), with the striped Tc-En expression. (D) In this Tc-btd RNAi embryo, the shape of germband (arrow) is abnormal, with the anterior regions including the head lobe being unidentified. However, it still expresses Tc-En as stripes. (D') The Tc-btd ${ }^{\text {RNAi }}$ embryo that remains intact with yolks before dissecting the germband (arrow) out. A trace of tissue (arrowhead) is seen in the anterior region of the embryo. Anterior is to the left. 
ously (Schinko et al., 2008). However, we find that Tc-btd is required for the development of the head and trunk in the later larval cuticles as well as in the early embryonic germbands, which was not revealed in the previous report (Schinko et al., 2008). This finding raises immediately two issues on the role of Tc-btd in Tribolium development.

The first issue is why the requirement of Tc-btd in the head development was not revealed in the previous study that utilized the parental and embryonic RNAi and confirmed significant knock-down of Tc-btd (Schinko et al., 2008). A reason for that could be the different genetic background of strains that has a major effect on the penetrance and severity of defects for the loss of a gene. Indeed, it has been reported that phenotypes generated with RNAi knock-down depend on the genotype of the used strain in Tribolium (Kitzmann et al., 2013). To knock down $T c$-btd, we utilized wild-type GA2 strain in the current study, whereas the wild-type stain used for knocking down $T c-b t d$ was not properly documented in the previous report (Schinko et al., 2008). Thus, the different genetic background of the wild-type strains used in the previous and current studies might be a reasonable explanation for the different conclusion on the Tc-btd function in head development. However, we could not completely rule out a possibility that potential off-target effects by knock-down of $T c-b t d$ may cause the defects in the head and trunk.

The second issue is on the effect of Tc-btd in the head and trunk development; is it necessary to pattern or shape the germbands? In order to shape the germband, first the germ rudiment forms with the condensation of blastomeres, and then extends its length fully through the combination of proliferation of cells at the posterior tip of the germ rudiment and the convergent extension of those cells (Brown et al., 1994; Cepeda et al., 2017). The head is patterned at the stage of blastoderm even before the germ rudiment forms, and the trunk is patterned at the stage of germband extension (Brown et al., 1994). The Tc-En stripes in the bifurcated head regions of the early growing
$T c-b t d^{\mathrm{RNAi}}$ germband suggest that somehow head patterning is achieved but the morphogenesis of the germband is disrupted. The short and wide germband with the Tc-En stripes in the fully extended $T c$-btd ${ }^{\mathrm{RNAi}}$ germband shows that germband growth is disrupted, whereas somehow the pattering of the trunk segmentation occurs. In addition, compared with other Tribolium segmentation genes that are expressed in the broad domains of the early blastoderm and in the posterior regions of the growing germbands (Choe et al., 2006; Choe \& Brown, 2007; Sarrazin et al., 2012; El-Sherif et al., 2012), Tc-btd seems to be expressed too late for patterning the head and trunk segmentation in the blastoderm and growing germbands, respectively. Based on the phenotypes of $T c-b t d^{\mathrm{RNAi}}$ germbands and the expression patterns of $T c-b t d$, we propose that $T c-b t d$ could be involved in shaping rather than in patterning the germbands at the stages of blastoderm and germband extension. In this context, the tissues of the abnormally shaped $T c$ $b t d^{\mathrm{RNAi}}$ germbands would not be patterned and survived properly in the later stages of head and trunk development, so would lead to the loss of the pregnathal and some abdominal segments in the $T c-b t d^{\mathrm{RNAi}}$ larvae. Comparative functional analysis of $b t d$ orthologs in other insects would reveal to what extent $b t d$ plays a role in patterning or morphogenesis for the development of early germbands.

\section{ACKNOWLEDGMENTS}

We thank Sujeong Kim, Minseok Kim, and Haeji Na for comments. This work was supported by Gyeongsang National University academic support (to H. J., J. O., and S. J.) and by Development Fund Foundation, Gyeongsang National University, 2015 (to C.P.C).

\section{REFERENCES}

Birkan M, Schaeper ND, Chipman AD (2011) Early patterning and blastodermal fate map of the head in the milk- 
weed bug Oncopeltus fasciatus. Evol Dev 13:436-447.

Brown SJ, Patel NH, Denell RE (1994) Embryonic expression of the single Tribolium engrailed homolog. Dev Genet 15:7-18.

Bucher G, Scholten J, Klingler M (2002) Parental RNAi in Tribolium (Coleoptera). Curr Biol 12:R85-86.

Cepeda RE, Pardo RV, Macaya CC, Sarrazin AF (2017) Contribution of cell proliferation to axial elongation in the red flour beetle Tribolium castaneum. PLOS ONE 12:e0186159.

Choe CP, Brown SJ (2007) Evolutionary flexibility of pairrule patterning revealed by functional analysis of secondary pair-rule genes, paired and sloppy-paired in the short-germ insect, Tribolium castaneum. Dev Biol 302:281-294.

Choe CP, Miller SC, Brown SJ (2006) A pair-rule gene circuit defines segments sequentially in the short-germ insect Tribolium castaneum. Proc Natl Acad Sci USA 103:6560-6564.

Chouard T (2008) Darwin 200: Beneath the surface. Nature 456:300-303.

Cohen SM, Jurgens G (1990) Mediation of Drosophila head development by gap-like segmentation genes. Nature 346:482-485.

Dalton D, Chadwick R, McGinnis W (1989) Expression and embryonic function of empty spiracles: A Drosophila homeo box gene with two patterning functions on the anterior-posterior axis of the embryo. Genes Dev 3: 1940-1956.

El-Sherif E, Averof M, Brown SJ (2012) A segmentation clock operating in blastoderm and germband stages of Tribolium development. Development 139:4341-4346.

Finkelstein R, Perrimon N (1990) The orthodenticle gene is regulated by bicoid and torso and specifies Drosophila head development. Nature 346:485-488.

Gao Q, Finkelstein R (1998) Targeting gene expression to the head: The Drosophila orthodenticle gene is a direct target of the Bicoid morphogen. Development 125:
4185-4193.

Kim EJ, Lee JM, Jung HS (2013) Fus expression patterns in developing tooth. Dev Reprod 17:215-220.

Kitzmann P, Schwirz J, Schmitt-Engel C, Bucher G (2013) RNAi phenotypes are influenced by the genetic background of the injected strain. BMC Genomics 14:5.

Lefort V, Longueville JE, Gascuel O (2017) SMS: Smart model selection in PhyML. Mol Biol Evol 34:24222424.

Patel NH, Condron BG, Zinn K (1994) Pair-rule expression patterns of even-skipped are found in both short- and long-germ beetles. Nature 367:429-434.

Rogers BT, Kaufman TC (1996) Structure of the insect head as revealed by the EN protein pattern in developing embryos. Development 122:3419-3432.

Rogers BT, Kaufman TC (1997) Structure of the insect head in ontogeny and phylogeny: A view from Drosophila. Int Rev Cytol 174:1-84.

Sarrazin AF, Peel AD, Averof M (2012) A segmentation clock with two-segment periodicity in insects. Science 336:338-341.

Schinko JB, Kreuzer N, Offen N, Posnien N, Wimmer EA, Bucher G (2008) Divergent functions of orthodenticle, empty spiracles and buttonhead in early head patterning of the beetle Tribolium castaneum (Coleoptera). Dev Biol 317:600-613.

Schroder R (2003) The genes orthodenticle and hunchback substitute for bicoid in the beetle Tribolium. Nature 422:621-625.

Sievers F, Wilm A, Dineen D, Gibson TJ, Karplus K, Li W, Lopez R, McWilliam H, Remmert M, Soding J, Thompson JD, Higgins DG (2011) Fast, scalable generation of high-quality protein multiple sequence alignments using Clustal Omega. Mol Syst Biol 7:539.

Snodgrass RE (1935) Principles of Insect Morphology. McGRaw Hill, New York.

St Johnston D, Nusslein-Volhard C (1992) The origin of pattern and polarity in the Drosophila embryo. Cell 
68:201-219.

Stauber M, Jackle H, Schmidt-Ott U (1999) The anterior determinant bicoid of Drosophila is a derived Hox class 3 gene. Proc Natl Acad Sci USA 96:3786-3789.

Walldorf U, Gehring WJ (1992) Empty spiracles, a gap gene containing a homeobox involved in Drosophila head development. EMBO J 11:2247-2259.

Wimmer EA, Frommer G, Purnell BA, Jackle H (1996) Buttonhead and D-Sp1: A novel Drosophila gene pair.
Mech Dev 59:53-62.

Wimmer EA, Jackle H, Pfeifle C, Cohen SM (1993) A Drosophila homologue of human $S p 1$ is a head-specific segmentation gene. Nature 366:690-694.

Wimmer EA, Simpson-Brose M, Cohen SM, Desplan C, Jackle H (1995) Trans- and cis-acting requirements for blastodermal expression of the head gap gene buttonhead. Mech Dev 53:235-245. 